collated, categorised, given a consequence score and recorded in this file. This allowed for a baseline to compare against future referrals.

Results The number of adverse events reported from the launch of BSW on 27 October 2008 until 30 January 2011 was 54. This compared to 100 adverse events reported during the time period 1 February 2011 to 27 November 2011. Referrals have increased fivefold since the intervention took place. Standardising the categories and consequences allowed for monitoring and comparison on the types of events reported to BSW per LAC. This helped to focus appropriate intervention including further education for reporting where it was needed. The type and severity of adverse events reported post intervention increased for the more minor and more serious adverse events. The less serious categories 1 and 2 saw almost a sevenfold increase in the rate of reporting while the more serious categories 3,4 and 5 experienced an increase of 1.5. There was concern that serious incidents went under-reported prior to the intervention. However, this evidence is re-assuring in that the rate of reporting is far higher for the more minor events post intervention than for the more serious events. The information for the more serious events was cross-checked retrospectively with the SSPs around Wales who confirmed these findings.

Conclusion The primary aim of the study was achieved and the annual rate of adverse events reporting to BSW has increased fivefold. Ongoing education is required to ensure adverse event reporting does not get forgotten and certainly until the process has become embedded in practice. The process and framework has enabled BSW to collate information providing a consistent approach for regular review and monitoring of adverse events by the BSW programme.

Competing interests None declared.

\section{PM0-011 EVALUATING PARTICIPANTS' EXPERIENCES OF THE BOWEL SCREENING WALES SERVICE}

doi:10.1136/gutjnl-2012-302514b.11

C Lewis.* Bowel Screening Wales, Public Health Wales, Llantrisant, UK

Introduction The aim of the study is to evaluate participants' opinions on their experience of the Bowel Screening Wales (BSW) service and thus provide a snapshot view of the programme.

Methods The primary research incorporated a thorough examination of peer-reviewed articles focusing on factors that may influence the patient experience of healthcare services. This included issues surrounding service performance, specifically, public satisfaction and experience processes, relationship with healthcare professionals, information giving and waiting times. Additionally region of residence and gender as influencing factors of user perspectives were discussed. An anonymous survey took the form of a selfadministered, postal questionnaire.

Results It was established that the users of the programme had a positive experience. User satisfaction was very high in all areas of the programme. Respondents were satisfied with the invitation pack and the way results were communicated to them. A high majority of those that contacted the freephone helpline were satisfied with the service they received. A high positive result was received in relation to satisfaction with the SSP appointment and the information received to prepare them for this appointment. $100 \%$ of respondents reported that they were satisfied with the services they received for further investigations. Participants appeared satisfied with the information provided to prepare them for a sensitive investigation which is significant since only one respondent had received a face to face appointment. The majority of respondents reported that they were treated with respect by the healthcare staff and that they were confident in their abilities. Longer waiting times did not appear to influence user satisfaction of the service. The data on region of residence and experience was inconclusive. It emerged from the data on sex and gender that there was little variation in the experiences of males and females. It is recognised that participants may respond to questionnaires in a favourable manner. However, the qualitative data provided by this study has confirmed the positive findings of the quantitative information.

Conclusion The outcomes of the study achieved the aims and objectives. Recommendations for service improvements were made to BSW based on the findings of this study. The study will allow for future snap shots to compare user experience and trend over time. This is a new national service and there were many comments expressing gratitude for the opportunity to participate and relief at obtaining a negative result. As the programme becomes embedded and users become more familiar with bowel screening there would be an opportunity to compare these finding against experiences in several years time.

Competing interests None declared.

\section{PM0-012 IMPROVING THE PATIENT JOURNEY IN HEPATOLOGY: THE EFFICACY OF PRE-APPOINTMENT INVESTIGATIONS}

doi:10.1136/gutjnl-2012-302514b.12

${ }^{1} \mathrm{C}$ Lever, ${ }^{*}{ }^{1} \mathrm{~J}$ House, ${ }^{2} \mathrm{~S}$ Ramaharack, ${ }^{3} \mathrm{~N}$ Sheron, ${ }^{3} \mathrm{M}$ Wright. ${ }^{1}$ Cost improvement and Transformation, University Hospital Southampton, Southampton, UK; ${ }^{2}$ Department of Medicine, Southampton University Hospital Trust, Southampton, UK; ${ }^{3}$ Department of Hepatology, Southampton University Hospital Trust, Southampton, UK

Introduction Most patients attending hepatology clinic for the first time require a basic liver screen before a definitive diagnosis or plan of action can be made. We sought to establish if doing these tests prior to the visit could speed up the patient journey.

Methods New referrals appraised by a consultant hepatologist and considered for routine outpatient appointments were entered in to the pre-appointment investigation study. 58 patients during July and August 2010 were sent blood forms and booked for ultrasound to be completed before coming to the first consultation. Of the 58 patients 55 were eligible for analysis.

Results 17 (30\%) patients had all blood results available at clinic, 30 (54\%) patients had imaging reports available and 10 (18\%) had both. At first consultation

Nine patients were discharged back to the GP

One booked for MRCP, discharged from clinic

One had no follow-up booked

Three discharged to the nurse led clinic

One booked into the virtual clinic

One went for TACE

$16(29 \%)$ of patients did not require a follow-up appointment in a consultant led hepatology clinic with 11 of these patients requiring no follow-up at all. 49 out of the 55 (89\%) patients had a definite diagnosis made at the first clinic appointment, verified subsequently by imaging and blood results. Compared to the same time frame for the year before a fivefold increase (from $4.3 \%$ to $20 \%$ ) in discharges to GP was achievable.

Conclusion This involves a commitment to arrange blood tests and imaging in advance, the costs are no different as these are routine investigations. With blood results available at the first consultation there was a significant increase in discharges. Simply changing the order of interventions results in a reduction in follow-up allocations, with an associated increase in the speed of patient progression across all clinics. Moving $89 \%$ of the patients seen in hepatology clinic on to the correct pathway at the first consultation, saves time and money.

Competing interests None declared. 